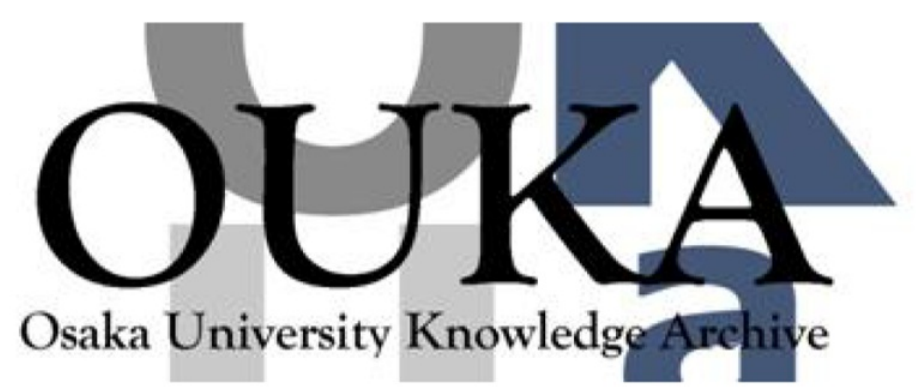

\begin{tabular}{|c|l|}
\hline Title & $\begin{array}{l}\text { Single-shot divergence measurements of a laser- } \\
\text { generated relativistic electron beam }\end{array}$ \\
\hline Author(s) & Perez, F.; Baton, S. D. ; Koenig, M. et al. \\
\hline Citation & $\begin{array}{l}\text { Physics of Plasmas. 17(11) p. 113106-1-p. 113106- } \\
7\end{array}$ \\
\hline Issue Date & $2010-11$ \\
\hline oaire:version & VoR \\
\hline URL & https://hdl. handle. net/11094/3392 \\
\hline rights & \\
\hline Note & \\
\hline
\end{tabular}

Osaka University Knowledge Archive : OUKA

https://ir. Library. osaka-u. ac. jp/

Osaka University 


\title{
Single-shot divergence measurements of a laser-generated relativistic electron beam
}

\author{
F. Perez, ${ }^{1, a)}$ S. D. Baton, ${ }^{1}$ M. Koenig, ${ }^{1}$ C. D. Chen, ${ }^{2}$ D. Hey, ${ }^{2}$ M. H. Key, ${ }^{2}$ S. Le Pape,${ }^{2}$ \\ T. $M a,{ }^{2}$ H. S. McLean, ${ }^{2}$ A. G. MacPhee, ${ }^{2}$ P. K. Patel, ${ }^{2}$ Y. Ping, ${ }^{2}$ F. N. Beg, ${ }^{3}$ \\ D. P. Higginson, ${ }^{3}$ C. W. Murphy, ${ }^{3}$ H. Sawada, ${ }^{3}$ B. Westover, ${ }^{3}$ T. Yabuuchi, ${ }^{3}$ K. U. Akli, ${ }^{4}$ \\ E. Giraldez, ${ }^{4}$ M. Hoppe, Jr., ${ }^{4}$ C. Shearer, ${ }^{4}$ R. B. Stephens, ${ }^{4}$ L. Gremillet, ${ }^{5}$ \\ E. Lefebvre, ${ }^{5}$ R. R. Freeman, ${ }^{6}$ G. E. Kemp, ${ }^{6}$ A. G. Krygier, ${ }^{6}$ L. D. Van Woerkom, ${ }^{6}$ \\ R. Fedosejevs, ${ }^{7}$ R. H. Friesen, ${ }^{7}$ Y. Y. Tsui, ${ }^{7}$ and D. Turnbull ${ }^{8}$ \\ ${ }^{1}$ Laboratoire pour l'Utilisation des Lasers Intenses, CNRS, CEA, UPMC, Ecole Polytechnique, \\ 91128 Palaiseau Cedex, France \\ ${ }^{2}$ Lawrence Livermore National Laboratory, Livermore, California 94550, USA \\ ${ }^{3}$ Department of Mechanical and Aerospace Engineering, University of California-San Diego, \\ La Jolla, California 92093, USA \\ ${ }^{4}$ General Atomics, San Diego, California 92121, USA \\ ${ }^{5}$ Commissariat à l'Énergie Atomique, DAM DIF, F-91297 Arpajon, France \\ ${ }^{6}$ College of Mathematical and Physical Sciences, The Ohio State University, Columbus, Ohio 43210, USA \\ ${ }^{7}$ Department of Electrical and Computer Engineering, University of Alberta, Edmonton, Alberta \\ T6G 2G7, Canada \\ ${ }^{8}$ Department of Mechanical and Aerospace Engineering, Princeton University, \\ Princeton, New Jersey 08544, USA
}

(Received 5 April 2010; accepted 21 October 2010; published online 9 November 2010)

\begin{abstract}
The relativistic electron transport induced by an ultraintense picosecond laser is experimentally investigated using an x-ray two-dimensional imaging system. Previous studies of the electron beam divergence [R. B. Stephens et al. Phys. Rev. E 69, 066414 (2004), for instance] were based on an $\mathrm{x}$-ray imaging of a fluorescence layer buried at different depths in the target along the propagation axis. This technique required several shots to be able to deduce the divergence of the beam. Other experiments produced single-shot images in a one-dimensional geometry. The present paper describes a new target design producing a single-shot, two-dimensional image of the electrons propagating in the target. Several characteristics of the electron beam are extracted and discussed and Monte Carlo simulations provide a good understanding of the observed beam shape. The proposed design has proven to be efficient, reliable, and promising for further similar studies.
\end{abstract}

(C) 2010 American Institute of Physics. [doi:10.1063/1.3514595]

\section{INTRODUCTION}

The concept of fast ignition (FI) (Ref. 1) applied to inertial confinement fusion requires a relativistic electron beam to heat the compressed fuel and ignite the fusion reactions. This beam is generated by a short (picosecond) ultraintense and high energy laser pulse interacting with hot dense matter. Knowledge of the electron beam generation and transport is of critical importance for the validation of the fast-ignition scheme. In addition, such relativistic electron beams are used in several other fields of physics such as high energy density physics (producing warm dense matter), ${ }^{2,3}$ particle acceleration, ${ }^{4}$ and ultrashort high energy x-ray sources. ${ }^{5-7}$

Many studies have been carried out to understand the transport of relativistic electrons in solid targets ${ }^{8,9}$ and compressed matter. ${ }^{10}$ One-dimensional (along electron beam propagation axis) measurements ${ }^{11-13}$ provide useful information on the energy distribution and laser-to-electron coupling efficiency but not on divergence features. Layered targets ${ }^{14,15}$ allow an extended characterization of the electron beam, but assume that shots with the same laser parameters produce the same electron beam. This assumption has been empirically

\footnotetext{
${ }^{a)}$ Electronic mail: frederic.perez@ polytechnique.edu.
}

found to be false and the shot-to-shot variation is a large source of error. ${ }^{16}$

In this article, a new target scheme is presented. It allows a direct measurement of the electron beam shape on a singleshot basis. Thus, this technique avoids the shot-to-shot uncertainty that is a huge source of errors. The experimental setup, the target design, and the diagnostics are described in Sec. II. The analysis method is detailed in Secs. III and IV. Numerical modeling is provided in Sec. V with the corresponding interpretation. Finally, an overall summary and conclusion is given in Sec. VI.

\section{EXPERIMENTAL SETUP}

The experiment was carried out on the Titan laser facility, providing a $150 \mathrm{~J}, 0.7 \mathrm{ps}$ pulse at the wavelength $1054 \mathrm{~nm}$, focused on a $10 \mu \mathrm{m}$ full width at half maximum (FWHM) spot containing approximately $30 \%$ of the total energy. The average laser intensity within this $10-\mu$ m-diameter region was approximately 8 $\times 10^{19} \mathrm{~W} / \mathrm{cm}^{2}$. The amplified spontaneous emission level before the main pulse and the prepulse intensity are reported by Le Pape et al. ${ }^{17}$ It typically contains $10 \mathrm{~mJ}$ within $3 \mathrm{~ns}$. 


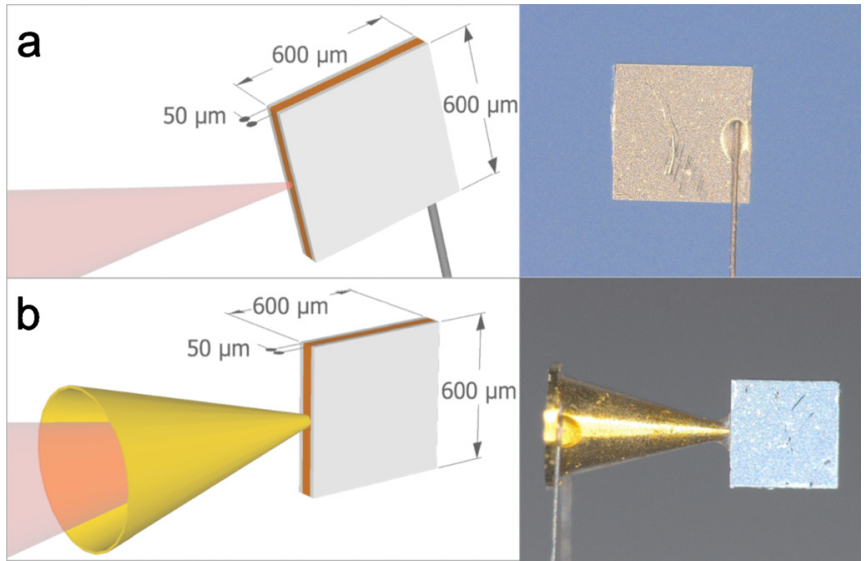

FIG. 1. (Color online) Target geometry schematics (a) without or (b) with a gold cone attached to the target edge. Corresponding photographs are given on the right-hand-side.

The targets were $600 \mu \mathrm{m} \times 600 \mu \mathrm{m}$ multilayer foils with $20 \mu \mathrm{m} \mathrm{Al}, 50 \mu \mathrm{m} \mathrm{Cu}$, and $20 \mu \mathrm{m} \mathrm{Al}$. The laser, instead of irradiating the large surface of the target, is focused on its edge, directly interacting with the copper layer as illustrated in Fig. 1(a). This feature constitutes the main difference with the usual multilayer target experiments. The $\mathrm{Al}$ layers on both sides of the $\mathrm{Cu}$ slab guaranteed that the $\mathrm{x}$-ray signal from the $\mathrm{Cu}$ atoms was not due to surface effects. To approach conditions close to the cone-guided FI scheme, a second type of target was used for which an empty gold cone was attached to the edge of the foil, as displayed in Fig. 1(b). The cones were $10 \mu \mathrm{m}$ thick with a $30-\mu \mathrm{m}$-wide flat tip and a $30^{\circ}$ opening angle. Note that to prevent light reflection back into the laser chain, the first kind of targets were slightly rotated downwards by $14^{\circ}$.

In order to infer the effect of electron refluxing inside the foil, smaller targets, $300 \mu \mathrm{m} \times 300 \mu \mathrm{m}$ wide, were also used. This refluxing corresponds to the electrons being reflected back inside the target when they reach one of its edges because of the strong subsequent surface electric fields. ${ }^{18}$ Smaller targets imply more refluxes for an electron of a given energy. Then the increased electron concentration is expected to induce a higher signal level.

The pointing accuracy of the laser was measured to be less than $\pm 10 \mu \mathrm{m}$. In the case of cone-guided targets, a dedicated back-reflection alignment diagnostic provided additional support.

Among the numerous diagnostics implemented, the most relevant one for this paper is the two-dimensional timeintegrated x-ray imaging system consisting of a spherically bent crystal reflecting the $\mathrm{Cu} K \alpha_{1}$ spectral line emission (8.048 keV x rays). ${ }^{19,20}$ The crystal was a quartz $21 \overline{3} 1$ with an interatomic distance $2 d=3.082 \AA$ and a $380 \mathrm{~mm}$ radius of curvature. The x-ray Bragg reflection on this curved crystal produces a 7.7-times magnified image with a Bragg angle of $88.7^{\circ}$. The detector was an imaging plate covered by a $12 \mu \mathrm{m} \mathrm{Al} \mathrm{filter.} \mathrm{As} \mathrm{shown} \mathrm{in} \mathrm{Fig.} \mathrm{2,} \mathrm{this} \mathrm{diagnostic} \mathrm{was}$ aligned for a transverse point of view i.e., side-on view. In our regime, most of the collected $\mathrm{x}$-ray signal is due to K-shell electron-impact ionization. The suprathermal elec-

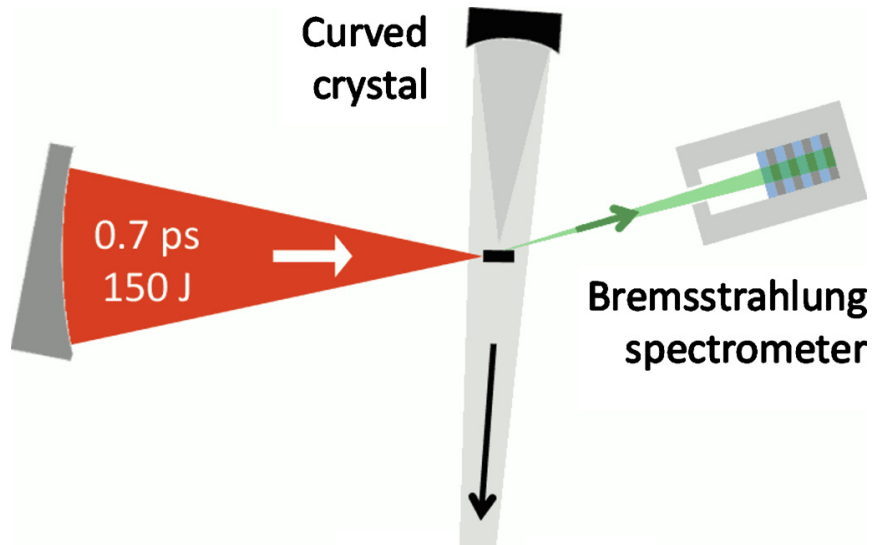

Ka X-rays image

FIG. 2. (Color online) Diagnostics layout.

trons generated in the laser interaction region efficiently ionize the $\mathrm{Cu}$ atoms in the $\mathrm{K}$-shell, and a subsequent atomic transition emits a $K \alpha$ photon. Therefore, the measured intensity of the $\mathrm{Cu} K \alpha$ spectral line is a direct signature of fast electrons propagating into the target bulk. The inner-shell ionization probability is quantified by the corresponding cross-section. ${ }^{21}$ The resulting emission is significant for electrons energies of $20 \mathrm{keV}$ or higher. Consequently, the thermal electron population is not detected by this instrument.

Bremsstrahlung spectrometers ${ }^{16}$ can also provide useful information about the electron beam energy distribution and shape. They consist of a stack of filters and imaging plates aligned toward the target, as shown in Fig. 2. The hard x-ray bremsstrahlung emission is successively attenuated by those filters and its energy is deposited in the different imaging plates. As the absorption for each filter is known, it is possible to determine the bremsstrahlung spectrum of the relativistic electron beam and thus infer the electron energy distribution. One bremsstrahlung spectrometer was used here to measure this electron energy spectrum. It was situated at the back of the target at a $16^{\circ}$ angle with the laser axis.

\section{X-RAY IMAGES ANALYSIS}

Figure 3(a) shows a sample image from the twodimensional x-ray imager. A bright spot originates from the laser interaction position, and shows that a fast electron

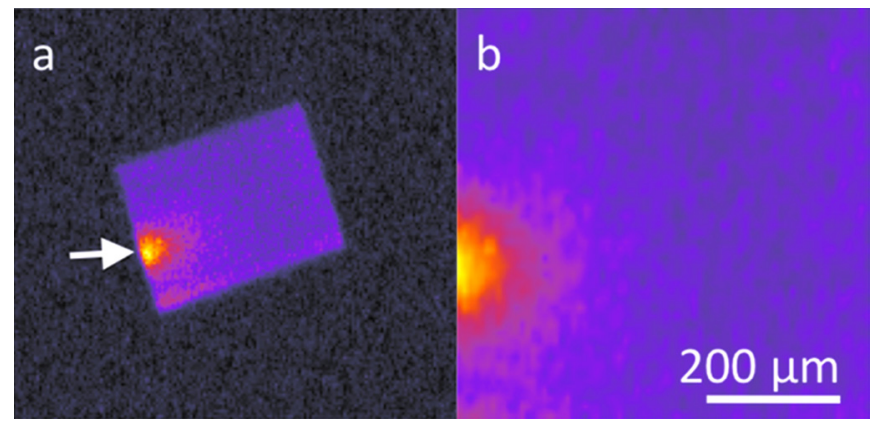

FIG. 3. (Color online) Side-view x-ray image of a $600 \times 600 \mu \mathrm{m}$ target without cone, raw (a), or reshaped (b). The arrow indicates the incoming laser direction. 


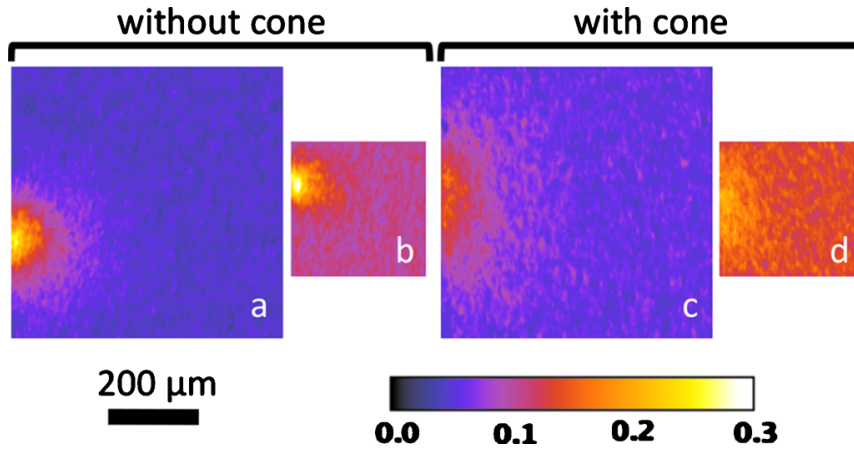

FIG. 4. (Color online) Typical images obtained without [(a) and (b)] or with [(c) and (d)] cone. (b) and (d) correspond to the smaller targets. The laser comes from the left-hand side and the color scale is in arbitrary units, although identical for all the displayed images.

beam is penetrating into the dense target up to at least $150 \mu \mathrm{m}$. To get rid of the distortion due to the viewing angle ( $40^{\circ}$ down from the equator plane), the image is reshaped as shown in Fig. 3(b). More precisely, we first apply a shear transformation to account for the target $14^{\circ}$ rotation, thus resulting in a rectangular image. Then, a vertical scaling compensates the inclination angle of the diagnostic, and produces the expected square-shaped image.

Note that, on the same images, a bright line is visible along the bottom edge of the target. This is simply due to the viewing angle of the detecting system: on that edge, the $\mathrm{Cu}$ layer is not covered by the $\mathrm{Al}$ foil. Thus, without any absorption from this Al foil, the signal appears brighter.

Typical results obtained during this experiment are displayed in Fig. 4. Each shot was repeated in order to check its consistency. The width of the electron beam close to the laser interaction region is about $100 \mu \mathrm{m}$, in agreement with the standard layered targets measurements. ${ }^{14}$ At first sight, we can see that the cone-guided targets yield a broader, less intense bright spot, but with a slightly higher background signal. The smaller targets present a much higher bulk signal level for an equal spot brightness, which can be attributed to the electron refluxing.

Important data can be extracted from the experimental images, such as the penetration depth and the beam divergence, which are the most useful ones for understanding electron transport. First of all, let us define the coordinate system describing the electron beam. Let $x$ be the axis of the laser and $y$ the perpendicular one. The origin $(0,0)$ is taken to be the center of the laser spot on the $\mathrm{Cu}$ edge.

Let us first consider the standard analysis carried out in multilayered target experiments. ${ }^{14}$ As illustrated in Fig. 5(a), it consists of taking the signal contained in a slice located at the depth $x_{0}$ and measuring the resulting spot FWHM $\Delta y$. For different depths $x_{0}$, the spot diameter $\Delta y$ varies. The corresponding slope gives an angle that is often said to be related to the beam divergence. ${ }^{15}$ Yet assigning this slope to an actual divergence is questionable as the measured angle is actually not simply related to the electron beam shape. To show this, let us assume an isotropic hot electron source. The fast-electron density $n_{h}(x, y)$ is then only a function of the radius $r=\sqrt{x^{2}+y^{2}}$ and can be written $n_{h}(r)$. We can take the
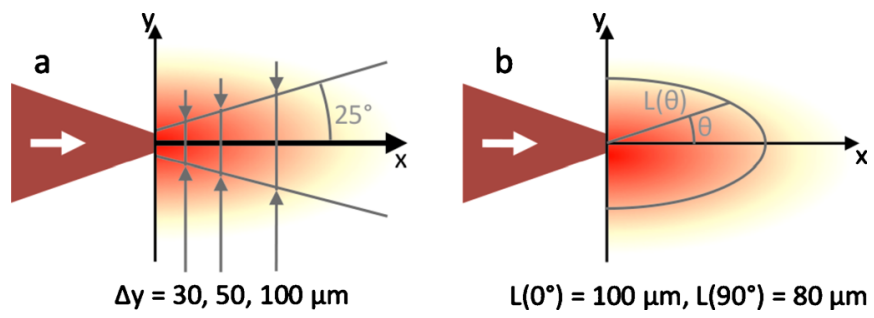

FIG. 5. (Color online) Illustration of the two different methods to analyze the same data, (a) as in layered targets experiments and (b) as in the present report.

example $n_{h}(r) \propto r^{-2}$. In the case of a fluorescent layer at the position $x_{0}$ inside the target, the $K \alpha$ spot intensity is described by $I(y) \propto n_{h}\left(x_{0}, y\right) \propto\left(x_{0}^{2}+y^{2}\right)^{-1}$ if the ionization crosssection does not depend much on the electron velocity. The spot diameter is the solution of $I(\Delta y / 2)=I(0) / 2$. We readily obtain $\Delta y=2 x_{0}$ hence yielding a $45^{\circ}$ half-opening angle. Recalling that this value was obtained assuming an isotropic electron beam, we have just shown that a simple measurement of a given "divergence" angle in a layered target cannot be easily linked to a beam preferentially propagating in one direction. We have therefore demonstrated that this procedure is not suited to accessing the angular properties of highly divergent electron beams, and thus should be avoided here.

An alternate analysis, illustrated in Fig. 5(b), can be employed to correctly interpret the experimental data. If the electron beam is isotropic, we can describe the $K \alpha$ images only using the radius $r$. The time-integrated intensity would be written as $I(r / L)$ where $L$ is a typical distance. However, one can imagine that the laser accelerates electrons preferentially along its propagation axis. In other words, the typical depth $L$ should be larger along the laser axis than in the other directions. This quantity $L$ must consequently depend on the angle coordinate $\theta$ defined as $\tan \theta=y / x$. Finally, an adequate expression of the signal intensity using the polar coordinates $(r, \theta)$ is $I[r / L(\theta)]$. Note that we implicitly supposed distances $r$ much larger than the laser spot size. In order to analyze the experimental data, we must assume an analytical expression for the two functions $I(r / L)$ and $L(\theta)$. For the former, the expression $I(r / L)=I_{0} \exp (-r / L)$ was found to be a good approximation of the experimental results. For the latter, the images from Fig. 4 show beams close to isotropic ones. Consequently we can choose a slowly varying depth versus angle, for example, $L(\theta)$ $=L_{0}\left(1-\alpha \sin ^{2} \theta\right)$ where $\alpha$ is a parameter determining the isotropy of the beam. Finally, an adequate approximation of the image in polar coordinates is

$$
I(r, \theta)=I_{0} \exp \left(\frac{-r / L_{0}}{1-\alpha \sin ^{2} \theta}\right) .
$$

To get a clearer idea of this beam shape, we can observe that the parameter $\alpha$ is equal to 0 for an isotropic beam, positive for a beam directed along the laser direction, and negative otherwise. Equivalently, one can define the more comprehensive parameter $\beta$ as the ratio between the forward typical 

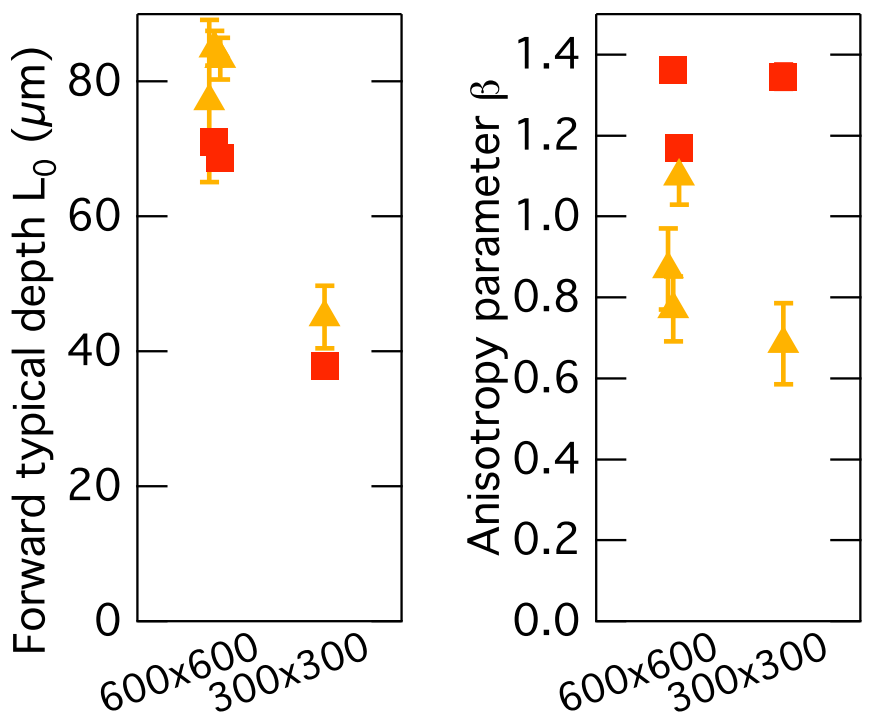

FIG. 6. (Color online) Results of the images analysis. The red squares and yellow triangles designate, respectively, the targets without and with cones.

depth $L\left(0^{\circ}\right)$ and the perpendicular typical depth $L\left(90^{\circ}\right)$. It translates into

$$
\beta=\frac{L\left(0^{\circ}\right)}{L\left(90^{\circ}\right)}=\frac{1}{1-\alpha} .
$$

This parameter is equal to 1 for an isotropic beam, greater than 1 for a cigar-shaped beam, and less than 1 for a pancake-shaped beam.

Each experimental image was successfully fitted with the ansatz from Eq. (1) using the three fit parameters $I_{0}, L_{0}$, and $\alpha$. The corresponding results are displayed in Fig. 6. The typical penetration depth is found to be about $70 \mu \mathrm{m}$, and slightly higher using cones. Surprisingly, small targets show a halved value, but this point will be discussed further. The anisotropy parameter $\beta$ is always above 1 without cones. As expected, it corresponds to an electron beam preferentially directed along the laser axis. However, for targets with cones, $\beta$ is lower and even below 1 . It means that electrons seem to go further when they are going perpendicularly to the laser axis, as suggested by the images from Fig. 4.

Obviously, this analysis does not rely on any assumption concerning the fast electron transport physics. Its aim is simply to provide better measured parameters than the usual layered targets divergence measurements. In order to extract physical phenomena, numerical simulations are needed.

\section{HARD X-RAY SPECTRA ANALYSIS}

Running electron transport simulations requires knowledge of the input hot-electron-beam divergence and energy distribution. To this goal, the bremsstrahlung spectrometer data was analyzed. The analysis method for this diagnostic is reported by Chen et al. ${ }^{16}$ It consists of modeling the electron stopping and bremsstrahlung emission using a collisional Monte Carlo code. By assuming an exponential energy distribution $d N / d E=\left(N_{0} / T_{0}\right) \exp \left(-E / T_{0}\right)$, the total number of electrons $N_{0}$, and their temperature $T_{0}$ can be inferred by finding the best fit to the experimental spectra. Note that, for

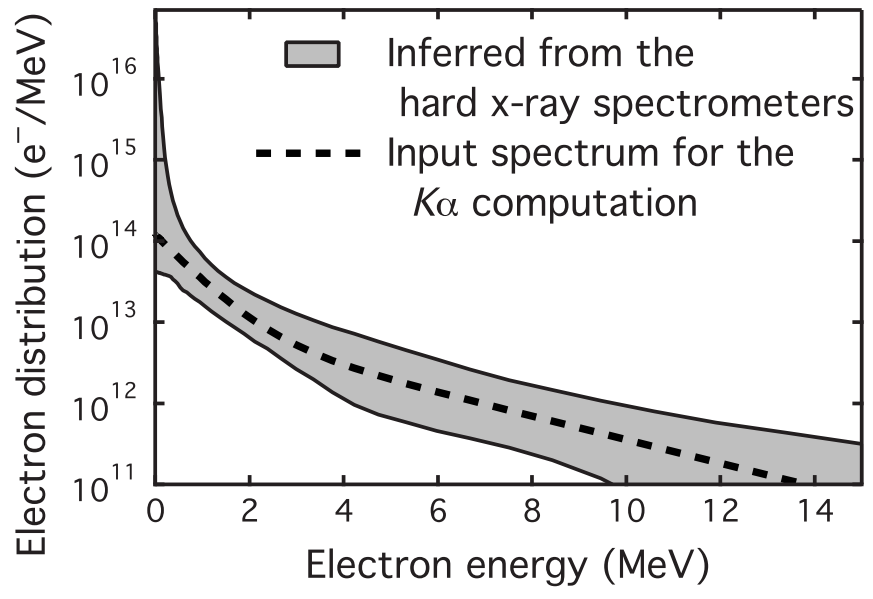

FIG. 7. Measured electron spectrum (gray area). The dashed line is the input spectrum used in Sec. V.

a better agreement with the experimental results, the sum of two distinct exponential distributions was used: $d N / d E$ $=\left(N_{0} / T_{0}\right) \exp \left(-E / T_{0}\right)+\left(N_{1} / T_{1}\right) \exp \left(-E / T_{1}\right)$.

While the targets used in this analysis are not identical to the targets described in the present paper, we do not expect the electron spectrum to be significantly modified, as only the surface conditions and the laser characteristics determine electron acceleration. The complete process is described in a separate, forthcoming publication. We present here the results relevant to our case. The best fitting range of electron energy distributions is displayed in Fig. 7 as a gray area. This spectrum is composed of exponential distributions with temperatures typically between 0.5 and $3 \mathrm{MeV}$. This measurement is used in Sec. V to compute the electron transport and $K \alpha$ images.

Note that, as the estimated electron energy spectrum is calculated for cone-free targets, it does not takes into account the modified surface conditions of cone-guided targets, and thus might not be valid in this case.

\section{V. $K \alpha$ IMAGES COMPUTATION}

In order to numerically reproduce the $K \alpha$ images, we used the Calder-MC Monte Carlo code. ${ }^{22}$ It includes the time-resolved $K \alpha$ computation with the ionization crosssection from Hombourger ${ }^{23}$ and the fluorescence yield from Bambynek. ${ }^{24}$ Electromagnetic fields are not taken into account. The target and the transport are fully threedimensional. Multiple scattering and slowing down are computed within a 2 fs time step for a few $10^{5}$ macroparticles in both the $\mathrm{Al}$ and $\mathrm{Cu}$ parts. The total duration of the simulations is $10 \mathrm{ps}$, and we verified the negligible contribution to the total $K \alpha$ emission of the remaining electrons at the end of the simulation.

This kind of simulation, which is similar to those used in conventional electron beam technologies, provides only an estimation of the electron transport after they are accelerated by the intense laser. The laser-plasma interaction is not computed as the complex phenomena involved require a very long computation time. Consequently, an input electron beam must be chosen (see next paragraph) and modified until 


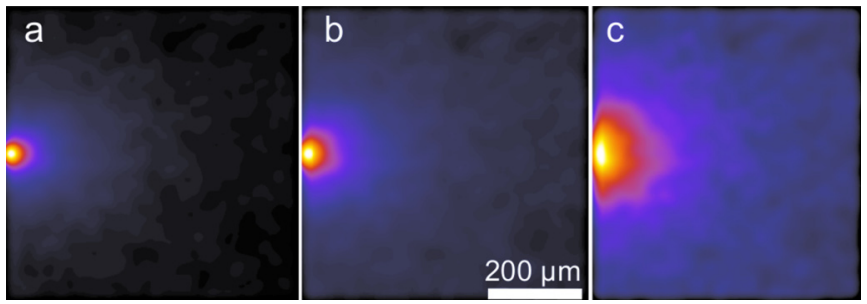

FIG. 8. (Color online) Simulated $K \alpha$ images (a) without preplasma, (b) with a $10 \mu \mathrm{m}$ preplasma, and (c) with a $50 \mu \mathrm{m}$ preplasma. The input halfopening divergence angle is $\theta_{0}=45^{\circ}$.

the results fit the experimental measurements. In other words, those simulations are only a tool for computing the electron transport from a given initial state. They are unable to predict the electron beam divergence.

The input electron spectrum is composed of two exponential distributions as indicated by the hard $\mathrm{x}$-ray spectrometers. Within the measured range from Fig. 7, the dashed line represents the chosen input electron spectrum. The two exponential distributions are defined by the total numbers of electrons $N_{0}=8 \times 10^{13}$ and $N_{1}=3 \times 10^{13}$ and the temperatures $T_{0}=700 \mathrm{keV}$ and $T_{1}=3 \mathrm{MeV}$, respectively. The input electron divergence is represented by a Gaussian angle distribution with a half-opening angle $\theta_{0}$ at half-maximum. In order to account for the refluxing, these electrons are specularly reflected when they reach any surface of the target.

A typical result is displayed in Fig. 8(a) for $\theta_{0}=45^{\circ}$. This computed x-ray image takes into account the opacity of $\mathrm{Cu}$ and $\mathrm{Al}$ for cold matter. The resulting $K \alpha$ spot radius turns out to be much smaller than the measured one [Fig. 4(a)]. Indeed, the depth $L_{0}$ is about $20 \mu \mathrm{m}$ instead of the measured $70 \mu \mathrm{m}$. In order to explain this significant difference, we tried several improvements to the $K \alpha$ computation.

As calculated by Akli et al., ${ }^{20}$ a high target temperature changes the atomic electron screening potential. As a result, the $K \alpha$ lines are eventually blueshifted outside the detector range. This implies a decreasing signal with increasing temperature. Thus, the hot and bright x-ray spot should emit less signal. In addition, the subsequent vacancies in the $L$-shell produce a bound-bound opacity for the same $K \alpha$ photons. Both effects were estimated using the atomic code FLYCHK. ${ }^{25}$ Those predicted opacity and emissivity variations with temperature were accounted for in the Calder-MC code. However, the resulting $K \alpha$ spot shape was not significantly modified because the heated region was not large enough. Indeed, only the first $5 \mu \mathrm{m}$ are heated above $200 \mathrm{eV}$. From the detector point of view, this volume is hidden behind $25 \mu \mathrm{m}$ of solid copper ( $70 \%$ opaque). The temperature gradient is not close enough to the target aluminum surface to have a significant effect.

The main cause for the large measured spot size is found to be a simple geometrical effect. The preplasma is expected to extend a few tens of microns in front of the target surface, thus modifying the electron source. ${ }^{26}$ According to hydrodynamic simulations using the HYDRA code, ${ }^{27}$ with the measured $2.3 \mathrm{~ns}$ pedestal containing $17 \mathrm{~mJ}$, the interaction region occurs 5-10 $\mu \mathrm{m}$ before the solid target surface. Furthermore, no x-ray emission can be detected from the preplasma as its density is too low and its temperature is too high (the $K \alpha$ lines are spectrally shifted outside the detector range). Consequently, the electron source has to be manually shifted by $\sim 10 \mu \mathrm{m}$ in the code. The corresponding result is given in Fig. 8(b) for a divergence half-angle $\theta_{0}=45^{\circ}$. The spot size, now typically $45 \mu \mathrm{m}$ long and with the anisotropy parameter $\beta=1.3$, fits better the experimental results. The electron beam, created away from the front surface, expands and slightly fades out before reaching the dense $\mathrm{Cu}$ plasma. This shows how important the preplasma size is in understanding $x$-ray images results. However, the experimental size of $70 \mu \mathrm{m}$ is still not completely explained by those simulations. However, the fields present in the preplasma will clearly play an important role. This was not taken into account here, and we can expect an increased spot size.

The role of the preplasma size is even clearer in the case of a cone-guided target, as evidenced in previous experiments with the support of Particle-In-Cell simulations. ${ }^{28,29}$ The HYDRA simulations show that the critical density is reached $50 \mu \mathrm{m}$ before the solid density $\mathrm{Cu}$ when a gold cone is attached to the target. In this case, the simulations lead to the x-ray image of Fig. 8(c), corresponding to the parameters $L_{0}=75 \mu \mathrm{m}$ and $\beta=0.9$. This agrees with the measurements shown in Fig. 6. It implies that the strongly diverging shape observed using cone-guided targets is mainly an artifact caused by the preplasma size. One should note that the preplasma-induced displacement of the source may not be the only effect on the observed electron beam shape. For instance, electromagnetic fields arising in the preplasma density gradient as well as corrugation of the gold surface can significantly modify the electron beam initial divergence. These effects cannot be reproduced with the presented simulations, and are beyond the scope of this paper.

As the simulated images are now in good agreement with the experimental ones, we can vary the divergence of the electron beam in order to check its influence on the $K \alpha$ spot shape. Several values of $\theta_{0}$ were tested and found to strongly modify the spot anisotropy. For instance, an input divergence $\theta_{0}=30^{\circ}$ leads to an anisotropy $\beta=1.6$, instead of 1.3 for $\theta_{0}=45^{\circ}$. Consequently, the measured value of $\beta$ is closely related to the electron beam divergence. In our case, we obtained the half-opening angle $\theta_{0}=40^{\circ} \pm 10^{\circ}$ for the best agreement with the experimental results, for both conefree and cone-guided targets.

Let us now consider the electron energy spectrum. Many electrons are slower than a few hundred keV. This implies that the imaging diagnostic will also detect the low energy (say $\lesssim 1 \mathrm{MeV}$ ) part of the electron spectrum. In order to estimate this contribution, Fig. 9 plots the fraction of $K \alpha$ photons created by electrons initially faster than $1 \mathrm{MeV}$. Far from the interaction region, most of the detected signal comes from the fastest electrons. Even within the bright $K \alpha$ spot, a significant part of the signal $(30 \%-70 \%)$ is due to the high energy electrons. Overall, the spot size is a combination of different electron energy components. The slowest electrons quickly take the form of an isotropic beam, causing the wide spot observed. The fastest electrons appear less influenced by collisional scattering, thus less divergent.

The contribution of the high energy part of the electron 

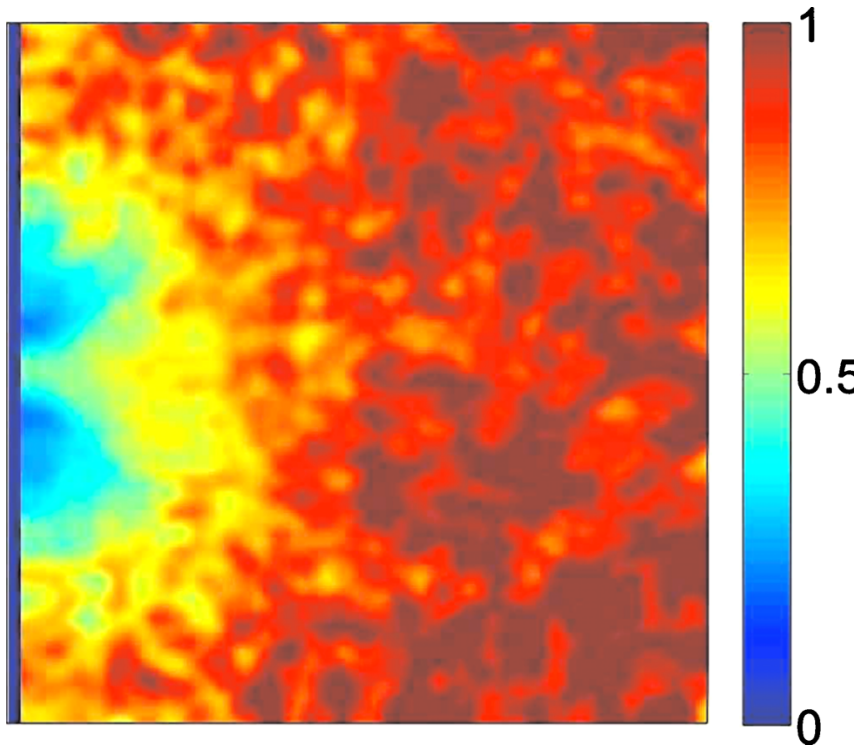

FIG. 9. (Color online) Simulated fraction of the $K \alpha$ emission produced by electrons initially faster than $1 \mathrm{MeV}$.

spectrum is confirmed by another feature. The uniform background signal level appeared significantly higher using reduced-size targets. The same behavior was numerically reproduced, the electron refluxing being represented as a specular reflection at the target surface. The small targets exhibit a five times higher background signal than the large targets. This confirms the presence of a high energy component in the electron spectrum.

\section{CONCLUSION}

The new target design presented in this article proved very effective in measuring the electron beam divergence. The experimental measurements have been interpreted by means of Monte Carlo simulations.

Three features appeared significant for understanding the beam shape. First, the electron refluxing at the target boundaries explained the high background signal level observed with small targets. Second, the slower electrons (typically $<1 \mathrm{MeV}$ ) undergo strong collisional effects inside the dense copper. Thus they quickly take the form of a quasi-isotropic beam. It explains why values of $\beta$ about 1.2 were obtained even though it seems to be rather low compared to the expected better collimation of the electron beam. Finally, the preplasma extension has to be included to reproduce correctly the beam shape. Indeed, cone-guided targets seemed to produce a strongly divergent electron beam. Simulations indicate that it is mostly a geometrical artifact due to the source being situated a few tens of microns outside the $\mathrm{Cu}$ target. Additional features are yet to be added to these simulations (as fields, a full preplasma shape, or a more realistic refluxing) for more precision on the observed results.

For a more complete understanding of the electron transport in solid targets, collisionless effects will be included in future studies. Using extended sets of experimental and numerical data, the influence of collective behaviors can be addressed. For example, magnetic fields arise from the cur- rent density gradients. In the present case, the current is not intense enough to generate a significant magnetic field deep inside the target. We do not expect it to greatly modify the overall electron beam shape, although it could be proved wrong with different conditions. Another collective feature to be studied is the electron slowing down caused by the return current energy loss via the Joule effect. This could change the stopping power value close to the electron source, and induce slight changes in the overall results. Different target or laser conditions could highlight these effects.

Overall, the electron beam anisotropy and penetration depth have been well characterized using a simple fitting function. This shape has been reproduced using a Monte Carlo code. The uniform intensity around the bright spot is due to higher energy electrons refluxing through the whole target, confirmed by the hard x-ray spectrometers. Most importantly, the input electron beam divergence significantly modified the computed x-ray spot shape, thus potentially leading to an absolute divergence measurement.

This new kind of solid-target geometry, used to measure two-dimensional fast-electron transport features, thus proved very efficient. Compared to multilayer targets, ${ }^{14,15}$ which often exhibit a strong shot-to-shot variation, single-shot measurements provide more precise data with a limited number of laser shots. Finally, these targets are an important step toward a better understanding of laser-induced electron transport in dense matter. These results lead the way for future studies aimed at measuring the electron divergence as a function of the target size and composition as well as the laser parameters or electromagnetic fields strength. One can also imagine more general studies with possible use of heating or compressing lasers in order to reach extreme states of matter closer to the fast-ignition conditions.

\section{ACKNOWLEDGMENTS}

The expert support of the Jupiter laser facility team, the Rutherford Appleton Laboratory, and the General Atomics target fabrication teams is gratefully acknowledged. This work was performed under the auspices of the U.S. Department of Energy by Lawrence Livermore National Laboratory under Contract No. DE-AC52-07NA27344.

\footnotetext{
${ }^{1}$ M. Tabak, J. Hammer, M. E. Glinsky, W. L. Kruer, S. C. Wilks, J. Woodworth, E. M. Campbell, M. D. Perry, and R. J. Mason, Phys. Plasmas 1, 1626 (1994).

${ }^{2}$ S. D. Baton, M. Koenig, P. Guillou, B. Loupias, A. Benuzzi-Mounaix, J. Fuchs, C. Rousseaux, L. Gremillet, D. Batani, A. Morace, M. Nakatsutsumi, R. Kodama, and Y. Aglitskiy, High Energy Density Phys. 3, 358 (2007).

${ }^{3}$ Y. Ping, A. Correa, T. Ogitsu, E. Draeger, E. Schwegler, T. Ao, K. Widmann, D. Price, E. Lee, H. Tam, P. Springer, D. Hanson, I. Koslow, D. Prendergast, G. Collins, and A. Ng, High Energy Density Phys. 6, 246 (2010).

${ }^{4}$ M. Roth, E. Brambrink, P. Audebert, A. Blazevic, R. Clarke, J. Cobble, T. Cowan, J. Fernandez, J. Fuchs, M. Geissel, D. Habs, M. Hegelich, S. Karsch, K. Ledingham, D. Neely, H. Ruhl, T. Schlegel, and J. Schreiber, Laser Part. Beams 23, 95 (2005).

${ }^{5}$ E. Brambrink, H. G. Wei, B. Barbrel, P. Audebert, A. Benuzzi-Mounaix, T. Boehly, T. Endo, C. Gregory, T. Kimura, R. Kodama, N. Ozaki, H. Park, M. R. le Gloahec, and M. Koenig, Phys. Plasmas 16, 033101 (2009).
} 
${ }^{6}$ H. Park, B. R. Maddox, E. Giraldez, S. P. Hatchett, L. T. Hudson, N. Izumi, M. H. Key, S. L. Pape, A. J. MacKinnon, A. G. MacPhee, P. K. Patel, T. W. Phillips, B. A. Remington, J. F. Seely, R. Tommasini, R. Town, J. Workman, and E. Brambrink, Phys. Plasmas 15, 072705 (2008). ${ }^{7}$ C. Courtois, A. C. L. Fontaine, O. Landoas, G. Lidove, V. Méot, P. Morel, R. Nuter, E. Lefebvre, A. Boscheron, J. Grenier, M. M. Aléonard, M. Gerbaux, F. Gobet, F. Hannachi, G. Malka, J. N. Scheurer, and M. Tarisien, Phys. Plasmas 16, 013105 (2009).

${ }^{8}$ P. Köster, K. Akli, D. Batani, S. Baton, R. G. Evans, A. Giulietti, D. Giulietti, L. A. Gizzi, J. S. Green, M. Koenig, L. Labate, A. Morace, P. Norreys, F. Perez, J. Waugh, N. Woolsey, and K. L. Lancaster, Plasma Phys. Controlled Fusion 51, 014007 (2009).

${ }^{9}$ D. Batani, S. Baton, M. Koenig, P. Guillou, B. Loupias, T. Vinci, C. Rousseaux, L. Gremillet, A. Morace, R. Redaelli, M. Nakatsutsumi, R. Kodama, N. Ozaki, T. Norimatsu, J. Rassuchine, T. Cowan, F. Dorchies, C. Fourment, and J. J. Santos, J. Phys.: Conf. Ser. 112, 022048 (2008).

${ }^{10}$ F. Perez, M. Koenig, D. Batani, S. D. Baton, F. N. Beg, C. Benedetti, E. Brambrink, S. Chawla, F. Dorchies, C. Fourment, M. Galimberti, L. A. Gizzi, R. Heathcote, D. P. Higginson, S. Hulin, R. Jafer, P. Koester, L. Labate, K. Lancaster, A. J. MacKinnon, A. G. McPhee, W. Nazarov, P. Nicolai, J. Pasley, A. Ravasio, M. Richetta, J. J. Santos, A. Sgattoni, C. Spindloe, B. Vauzour, and L. Volpe, Plasma Phys. Controlled Fusion 51, 124035 (2009).

${ }^{11}$ T. Ma, M. H. Key, R. J. Mason, K. U. Akli, R. L. Daskalova, R. R. Freeman, J. S. Green, K. Highbarger, P. A. Jaanimagi, J. A. King, K. L. Lancaster, S. P. Hatchett, A. J. Mackinnon, A. G. MacPhee, P. A. Norreys, P. K. Patel, R. B. Stephens, W. Theobald, L. D. V. Woerkom, M. S. Wei, S. C. Wilks, and F. N. Beg, Phys. Plasmas 16, 112702 (2009).

${ }^{12}$ J. A. King, K. U. Akli, R. R. Freeman, J. Green, S. P. Hatchett, D. Hey, P. Jamangi, M. H. Key, J. Koch, K. L. Lancaster, T. Ma, A. J. MacKinnon, A. MacPhee, P. A. Norreys, P. K. Patel, T. Phillips, R. B. Stephens, W. Theobald, R. P. J. Town, L. V. Woerkom, B. Zhang, and F. N. Beg, Phys. Plasmas 16, 020701 (2009).

${ }^{13}$ R. Kodama, Y. Sentoku, Z. L. Chen, G. R. Kumar, S. P. Hatchett, Y. Toyama, T. E. Cowan, R. R. Freeman, J. Fuchs, Y. Izawa, M. H. Key, Y. Kitagawa, K. Kondo, T. Matsuoka, H. Nakamura, M. Nakatsutsumi, P. A. Norreys, T. Norimatsu, R. A. Snavely, R. B. Stephens, M. Tampo, K. A. Tanaka, and T. Yabuuchi, Nature (London) 432, 1005 (2004).

${ }^{14}$ R. B. Stephens, R. A. Snavely, Y. Aglitskiy, F. Amiranoff, C. Andersen, D. Batani, S. D. Baton, T. Cowan, R. R. Freeman, T. Hall, S. P. Hatchett, J. M. Hill, M. H. Key, J. A. King, J. A. Koch, M. Koenig, A. J. MacKinnon, K. L. Lancaster, E. Martinolli, P. Norreys, E. Perelli-Cippo, M. R. L. Gloahec, C. Rousseaux, J. J. Santos, and F. Scianitti, Phys. Rev. E 69, 066414 (2004).

${ }^{15}$ J. S. Green, V. M. Ovchinnikov, R. G. Evans, K. U. Akli, H. Azechi, F. N. Beg, C. Bellei, R. R. Freeman, H. Habara, R. Heathcote, M. H. Key, J. A. King, K. L. Lancaster, N. C. Lopes, T. Ma, A. J. MacKinnon, K. Markey, A. McPhee, Z. Najmudin, P. Nilson, R. Onofrei, R. Stephens, K. Takeda,
K. A. Tanaka, W. Theobald, T. Tanimoto, and J. Waugh, Phys. Rev. Lett. 100, 015003 (2008).

${ }^{16}$ C. D. Chen, P. K. Patel, D. S. Hey, A. J. Mackinnon, M. H. Key, K. U. Akli, T. Bartal, F. N. Beg, S. Chawla, H. Chen, R. R. Freeman, D. P. Higginson, A. Link, T. Y. Ma, A. G. MacPhee, R. B. Stephens, L. D. V. Woerkom, B. Westover, and M. Porkolab, Phys. Plasmas 16, 082705 (2009).

${ }^{17}$ S. Le Pape, Y. Y. Tsui, A. Macphee, D. Hey, P. Patel, A. Mackinnon, M. Key, M. Wei, T. Ma, F. N. Beg, R. Stephens, K. Akli, T. Link, L. Van-Woerkom, and R. R. Freeman, Opt. Lett. 34, 2997 (2009).

${ }^{18}$ E. E. Fill, Phys. Plasmas 12, 052704 (2005).

${ }^{19}$ J. A. Koch, Y. Aglitskiy, C. Brown, T. Cowan, R. Freeman, S. Hatchett, G. Holland, M. Key, A. MacKinnon, J. Seely, R. Snavely, and R. Stephens, Rev. Sci. Instrum. 74, 2130 (2003).

${ }^{20}$ K. U. Akli, M. H. Key, H. K. Chung, S. B. Hansen, R. R. Freeman, M. H. Chen, G. Gregori, S. Hatchett, D. Hey, N. Izumi, J. King, J. Kuba, P. Norreys, A. J. Mackinnon, C. D. Murphy, R. Snavely, R. B. Stephens, C. Stoeckel, W. Theobald, and B. Zhang, Phys. Plasmas 14, 023102 (2007).

${ }^{21}$ M. Talukder, S. Bose, and S. Takamura, Int. J. Mass. Spectrom. 269, 118 (2008).

${ }^{22}$ E. Lefebvre, N. Cochet, S. Fritzler, V. Malka, M.-M. Aléonard, J.-F. Chemin, S. Darbon, L. Disdier, J. Faure, A. Fedotoff, O. Landoas, G. Malka, V. Méot, P. Morel, M. Rabec Le Gloahec, A. Rouyer, Ch. Rubbelynck, V. Tikhonchuk, R. Wrobel, P. Audebert, and C. Rousseaux, Nucl. Fusion 43, 629 (2003).

${ }^{23}$ C. Hombourger, J. Phys. B 31, 3693 (1998).

${ }^{24} \mathrm{~W}$. Bambynek, in X-Ray and Inner-Shell Processes in Atoms, Molecules and Solids, edited by A. Meisel and J. Finster (Karl-Marx-Universität, Leipzig, 1984).

${ }^{25}$ H. Chung, M. Chen, W. Morgan, Y. Ralchenko, and R. Lee, High Energy Density Phys. 1, 3 (2005).

${ }^{26}$ F. Perez, L. Gremillet, M. Koenig, S. D. Baton, P. Audebert, M. Chahid, C. Rousseaux, M. Drouin, E. Lefebvre, T. Vinci, J. Rassuchine, T. Cowan, S. A. Gaillard, K. A. Flippo, and R. Shepherd, Phys. Rev. Lett. 104, 085001 (2010).

${ }^{27}$ M. M. Marinak, G. D. Kerbel, N. A. Gentile, O. Jones, D. Munro, S. Pollaine, T. R. Dittrich, and S. W. Haan, Phys. Plasmas 8, 2275 (2001).

${ }^{28}$ S. D. Baton, M. Koenig, J. Fuchs, A. Benuzzi-Mounaix, P. Guillou, B. Loupias, T. Vinci, L. Gremillet, C. Rousseaux, M. Drouin, E. Lefebvre, F. Dorchies, C. Fourment, J. J. Santos, D. Batani, A. Morace, R. Redaelli, M. Nakatsutsumi, R. Kodama, A. Nishida, N. Ozaki, T. Norimatsu, Y. Aglitskiy, S. Atzeni, and A. Schiavi, Phys. Plasmas 15, 042706 (2008).

${ }^{29}$ A. G. MacPhee, L. Divol, A. J. Kemp, K. U. Akli, F. N. Beg, C. D. Chen, H. Chen, D. S. Hey, R. J. Fedosejevs, R. R. Freeman, M. Henesian, M. H. Key, S. L. Pape, A. Link, T. Ma, A. J. Mackinnon, V. M. Ovchinnikov, P. K. Patel, T. W. Phillips, R. B. Stephens, M. Tabak, R. Town, Y. Y. Tsui, L. D. V. Woerkom, M. S. Wei, and S. C. Wilks, Phys. Rev. Lett. 104, 055002 (2010). 